\title{
Una conversación entre la filosofía analítica y el psicoanálisis: sobre The Unconscious, de Alasdair MacIntyre*
}

\section{A CONVERSATION BETWEEN ANALYTIC PHILOSOPHY AND PSYCHOANALYSIS: ON THE UNCONSCIOUS, BY ALASDAIR MACINTYRE}

Carlos de la Puente Arbaiza

Universidad de Lima

capuente@ulima.edu.pe

\section{RESUMEN}

En este artículo se analiza la obra de Alasdair MacIntyre, The Unconscious. Se postula que este libro interroga la teoría psicoanalítica desde la perspectiva de la filosofía analítica de la mente y continúa así la conversación entre el psicoanálisis y la filosofía anglosajona. Se discuten las tesis de Maclntyre en el sentido de que el concepto de inconsciente no es necesario para formular leyes científicas que correlacionen experiencias de la infancia con la vida adulta, y se concluye que, al plantear esta tesis, MacIntyre ignoró lo que él mismo considera que es el mayor logro de la teoría psicoanalítica.

PALABRAS CLAVE: psicoanálisis, filosofía analítica, Wittgenstein, Freud, inconsciente, causalidad, razones
ABSTRACT

This article analyzes Alasdair Maclntyre's The Unconscious. This book interrogates the psychoanalytic theory from the analytic philosophy of mind and thus continues the conversation between psychoanalysis and Anglo-Saxon philosophy. Maclntyre's thesis is discussed, namely, that the concept of the unconscious is not necessary to formulate scientific laws that correlate childhood experiences with adult life, concluding that, MacIntyre ignored, when raising this thesis, what he himself considers to be the greatest achievement of psychoanalytic theory.

KEYWORDS: psychoanalysis, analytic philosophy, Wittgenstein, Freud, unconscious, causality, reasons

\footnotetext{
* Este artículo se empezó a elaborar como parte de una investigación apoyada por el Instituto de Investigación Científica de la Universidad de Lima (IDIC).
} 
La relación del psicoanálisis con la filosofía ha sido tensa y ambivalente. Ha habido cuestionamientos, sobre todo desde la filosofía al psicoanálisis, pero también se ha producido un intercambio productivo. Por el lado de las críticas, Freud fue el primero en declarar las hostilidades. En reiteradas ocasiones y desde sus primeras obras condenó a la filosofía por limitar lo mental a lo consciente y desconocer, por lo tanto, lo inconsciente. En algún escrito llegó a trazar una equivalencia entre el modo de razonar de los filósofos con el de los psicóticos. No es muy halagador para los filósofos ${ }^{1}$. Desde la filosofía se ha reprochado al psicoanálisis la falta de claridad conceptual y la imposibilidad de corroborar sus hipótesis, y, consecuentemente, la ausencia de sustento empírico para sus teorías. Pero junto a estos cuestionamientos ha existido también una relación de cooperación. En el psicoanálisis, después de Freud, han surgido escuelas que incorporaron en sus teorías ideas desarrolladas en el ámbito de la filosofía. La escuela psicoanalítica intersubjetiva es un ejemplo de esto. La filosofía, por su parte, ha utilizado ideas surgidas en el psicoanálisis para tratar de encontrar respuestas a algunas de sus preguntas. En relación con el antiguo problema de la irracionalidad, por ejemplo, Donald Davidson, David Pears y Mark Johnston, entre otros autores, han echado mano de nociones surgidas en Freud para dilucidar este rompecabezas que desde Platón y Aristóteles causa perplejidad. En otro campo de la filosofía, el de la ética y la teoría social, Axel Honneth (2009) ha dicho que la idea de un potencial para la acción que ha escapado a los procesos de socialización (que es como podría entenderse al inconsciente) es indispensable para una concepción "intersubjetiva" de la persona. Esta concepción, que según este influyente autor permitirá una noción razonable, es decir, poskantiana, de autonomía personal, solo puede alcanzarse con una teoría intersubjetiva del sujeto “ampliada por el psicoanálisis" (p. 279).

Son por lo menos tres corrientes en la filosofía del siglo xx en las que ha habido una aproximación sistemática al psicoanálisis: en la fenomenología existencial, con Merleau Ponty y Sartre; en la Escuela de Fráncfort; y en la filosofía anglosajona, con el pragmatismo y la filosofía analítica de la mente.

La conversación entre el psicoanálisis y la filosofía anglosajona comenzó con artículos de Bertrand Russell y John Dewey, y con apuntes rápidos pero influyentes de Ludwig Wittgenstein. Pero mientras la discusión de las ideas psicoanalíticas llevadas a cabo por Russell, Dewey y Wittgenstein se plasmaron en capítulos o comentarios dentro de trabajos de alcance más general, Alasdair MacIntyre (nacido en 1929) publicó en 1958 el primer libro dedicado íntegramente a interpretar las ideas psicoanalíticas desde la

1 "Cuando pensamos en abstracto nos exponemos al peligro de descuidar vínculos de las palabras con las representaciones, cosa inconsciente, y es innegable que, entonces, nuestro filosofar cobra una indeseada semejanza, en su expresión y contenido, con la modalidad de trabajo de los esquizofrénicos" (Freud, 1915/1979a, p. 200) 
perspectiva de la filosofía analítica de la mente, The Unconscious. A Conceptual Analysis (Routledge, 2004). Este es un trabajo corto que reconoce el aporte genial de Freud a las ciencias humanas y que expresa, además, convicción acerca del poder curativo de la terapia psicoanalítica, al mismo tiempo que señala confusiones conceptuales en el pensamiento de Freud.

Maclntyre cree que el marco conceptual para entender la teoría psicoanalítica es el aristotelismo. Una de las cosas que le impide a un ser humano alcanzar el ideal de una vida racional (como concibió Aristóteles este ideal) es vivir dominado por deseos mal concebidos, deseos generados por sentimientos y fantasías exageradas. Asustarse o sentir fuerte ansiedad por cosas que en verdad no son amenazantes o sentir indiferencia por situaciones que sí son peligrosas son ejemplos - dice este autor-de juicios prácticos equivocados. Por esta razón, una comprensión psicoanalítica del deseo y de la fantasía es necesaria para entender y poder superar los obstáculos que interfieren con el logro de una vida racional. Esto, lógicamente, tiene implicancias para la filosofía política porque una teoría sobre una sociedad justa, que para un aristotélico como Maclntyre es una comunidad en la que los ciudadanos pueden desarrollar sin barreras su potencial como seres humanos, debe incorporar las enseñanzas del psicoanálisis acerca de las ataduras internas -emocionales, podríamos decir-que impiden la autorrealización de los individuos. Una teoría política que no tome en cuenta los insights psicoanalíticos es, por lo tanto, "seriamente defectuosa", del mismo modo que una comprensión psicoanalítica que no está integrada a (y no es complementada por) una filosofía moral y política es gravemente defectuosa (Maclntyre, 2004, p. 27)².

Se puede estar en desacuerdo con MacIntyre cuando dice que la filosofía aristotélica es la que mejor ha articulado en la tradición occidental el ideal de una vida racional, pero no se puede negar, en cambio, que el concepto de cura psicoanalítica necesita de un ideal normativo que lo haga más claro. $Y$ tampoco se puede negar que el ideal normativo del psicoanálisis, independientemente de si es formulado bajo el canon de la filosofía práctica de Aristóteles, como lo propone Maclntyre, tiene que referirse a los ideales de la libertad y la racionalidad. La terapia psicoanalítica existe para ayudar a las personas a recuperar su libertad interna.

Maclntyre declara que su interés en este libro es integrar la teoría psicoanalítica con la filosofía analítica de la mente de Gilbert Ryle, pero la influencia de Wittgenstein

2 Otro punto de colaboración entre el psicoanálisis y la filosofía moral, relacionado con el que Maclntyre señala, es el estudio del problema de la reificación y el de la alienación. El tratamiento que le han dado a estos problemas autores de la Escuela de Fráncfort como Axel Honneth (a la reificación) y Rainer Forst (al de la alienación) constituye una invitación a una colaboración interdisciplinaria de la filosofía con el psicoanálisis. Esto ya se encuentra en el trabajo de Honneth; en cambio, no en el de Forst, cuya comprensión del fenómeno de la alienación (la alienación noumenal, como la llama) puede enriquecerse con un aporte del psicoanálisis. 
es notoria también. Maclntyre postula, siguiendo lo que dice Wittgenstein en El libro azul, que un deseo inconsciente es aquel que, al tiempo de ser el móvil de una conducta, no es expresado verbalmente por la persona. Esto quiere decir que cuando actúo motivado por un deseo inconsciente, hay una discrepancia entre lo que hago y lo que digo como explicación a mi conducta. Si me preguntan "¿por qué haces eso?" cuando mi motivación es inconsciente, mi respuesta será involuntaria y necesariamente falsa, porque una suerte de interferencia o cortocircuito en mi mundo interno me impide conocer y articular mis verdaderos motivos.

La tesis de Maclntyre es que Freud fue víctima de confusión conceptual en la formulación de este hallazgo. Esta tesis tiene dos partes que están relacionadas: la primera es que Freud no diferenció entre motivos y causas de una conducta; la segunda es que el concepto de inconsciente, como lo plantea el psicoanálisis, es útil para la descripción de las conductas, pero prescindible para la explicación. De acuerdo a la segunda parte de la tesis, las relaciones causales entre experiencias infantiles y rasgos de la personalidad en los adultos (sobre las que el psicoanálisis ha insistido más que ninguna otra corriente de la psicología) pueden establecerse sin necesidad de invocar el concepto de inconsciente.

Sobre la primera parte, el logro más importante de Freud, según Maclntyre, fue descubrir para la psicología una nueva manera de observar las acciones de los seres humanos. Gracias a esta nueva mirada, Freud encontró motivos e intenciones en acciones $^{3}$ cuyo propósito nadie antes que él había podido discernir. Freud reveló que el ritual del obsesivo, las fantasías diurnas, los sueños, algunos olvidos, así como los lapsus del habla y de la escritura, entre otras conductas, tienen una finalidad. Hay algo que las personas quieren decir y hacer a través de estos actos. El revolucionario hallazgo del psicoanálisis consiste, por lo tanto, en haber descubierto una dimensión teleológica, un sentido en conductas que antes parecían erráticas.

Incluso algunos de los novelistas más conscientes de la opacidad de la realidad humana, como Marcel Proust y Henry James, representaron las sombras del malentendido en contraposición a una luz de entendimiento. Pero Freud sistemáticamente explora lo misterioso, lo que hasta para él parecía incomprensible, y lo anormal de la vida humana, en tal grado que altera completamente los límites entre lo comprensible y lo incomprensible. (Maclntyre, 2004, p. 91)

La afirmación de MacIntyre es que Freud no habría entendido cabalmente su propio hallazgo, ya que se refirió a los deseos inconscientes de los neuróticos (como los mencionados en el párrafo anterior) como "causas" de las conductas, cuando en realidad se

3 Es gracias al psicoanálisis que hemos ampliado el rango de lo que la palabra acciones cubre. Porque ahora sabemos que, por ejemplo, una parálisis corporal que no tenga causas orgánicas puede ser también una manera de actuar. 
trata de razones para la acción o, lo que viene a ser lo mismo, de propósitos, intenciones o motivos.

La diferencia entre razones y causas ha sido tratada por varios filósofos, especialmente Kant (1997), cuando afirmó que la libertad es una causalidad, pero "de una clase especial" (p. 63). Las acciones de los seres humanos están dirigidas por razones, es decir, por propósitos que pueden ser explicados por la persona que actúa. Son motivos que, desde la perspectiva del agente, confieren racionalidad a una acción. En cambio, las causas existen en el mundo natural, por lo que se suele hablar de "fuerzas ciegas" para referirse a los eventos naturales que actúan como causas. La visión dominante en la filosofía analítica de la mente fue, hasta los escritos de Donald Davidson, que la explicación de las acciones intencionales de los seres humanos debía prestar atención únicamente a las razones. De acuerdo con esta concepción, no cabría hablar de causas en relación con las acciones inteligentes, porque la relación causal es contingente y no ha sido decidida por ninguno de los eventos que participan en esta relación. En la conducta de los humanos, en cambio, es esencial la decisión que la persona toma sobre la secuencia de sus acciones.

Este punto de vista, que expresa que las explicaciones en psicología deben ser teleológicas o interpretativas, fue desarrollado por Wittgenstein precisamente en sus críticas al psicoanálisis y fue asumido por varias generaciones de filósofos analíticos. Así, Elizabeth Anscombe sostiene en su libro Intention (1957) que en la explicación de las acciones intencionales se debe considerar solo las razones. Ella no niega la existencia de una causalidad en los fenómenos mentales (mental causality), pero cuando se trata de los sentimientos y de las acciones, que son los hechos mentales que interesan a los psicoanalistas, los factores causales explican bastante menos - si acaso- que las razones. Así, en los sentimientos, dice Anscombe siguiendo a Wittgenstein, hay que diferenciar entre el objeto del sentimiento, es decir, las razones que justifican un sentimiento, y su causa. Yo puedo estar molesto por lo que una persona dijo, y el contenido de la observación hecha por aquella persona sería el objeto de mi molestia, pero la causa de mi molestia será el instante en que algo o alguien me recuerden lo que esa persona dijo. Un niño puede asustarse frente a un trapo rojo cuando su hermano mayor, de manera maliciosa, le dice que ese trapo rojo es un pedazo de Satán. La causa del miedo del niño es el evento que tiene lugar cuando el hermano mayor hace ese comentario malvado, pero el objeto del miedo, aquello a lo que el miedo está dirigido, es el trapo y su significado. Anscombe dice que algunas acciones responden más a factores causales, como cuando un ladrido fuerte me hace soltar los vasos que estaba cargando, y otras son claramente motivadas por razones, como cuando actúo por venganza, por celos o por compasión. El criterio para diferenciar causas de razones es que uno puede criticar las razones y no así las causas. Tiene poco sentido decirle a alguien que no debe reaccionar de esa manera frente a un ladrido fuerte, pero sí tiene sentido decirle que los celos o la compasión no 
son razones suficientes para tal o cual acción. La línea que separa las razones de las causas, en el comportamiento de los seres humanos y en los sentimientos, no es nítida.

En general, mientras más uno describa una acción como una respuesta, uno va a estar más inclinado a usar la palabra causa; en tanto que mientras más uno describa una acción como una respuesta a algo teniendo un significado que es ponderado por el agente en la descripción de su acción, o una respuesta rodeada de pensamientos y preguntas, uno va a estar más inclinado a usar la palabra razón. (Anscombe, 1957, p. 23)

Del mismo modo con los sentimientos. El objeto de un sentimiento es algo constituido por pensamientos y valoraciones. La causa, en cambio, es cualquier evento que desencadena los pensamientos que forman parte del objeto del sentimiento.

Como Anscombe y la mayoría de los filósofos analíticos de la época en que Maclntyre escribió The Unconscious, este autor asumió esta distinción tajante entre causas y razones. Asimismo, sostuvo que los deseos inconscientes son fundamentalmente razones; tienen también un elemento causal, pero para él Freud colocó en el lugar equivocado este factor causal.

Pace Maclntyre, esta crítica solo es válida si pensamos en los escritos epistemológicos o teóricos de Freud, en los que, ciertamente y por lo general, se le da al deseo inconsciente el tratamiento que corresponde a una causa. El no distinguir entre causa y razón fue, por lo demás, común a casi todos los filósofos y psicólogos hasta que esa distinción llegó a la filosofía psicológica a través de Wittgenstein. En el caso de Freud, como en el de otros, no fue una confusión solamente terminológica; en algunos de estos escritos teóricos ignoró todo elemento intencional en los impulsos amorosos y agresivos, y llegó a pensar sobre ellos casi como equivalentes a descargas eléctricas.

En cambio, la crítica no es válida si pensamos en los escritos de casos clínicos en los que Freud consideró los deseos inconscientes de los neuróticos como razones. El título de una de sus conferencias, "El sentido de los síntomas" (1917), y su contenido no admiten dudas sobre eso. En ese trabajo afirmó que los neuróticos que actúan bajo la compulsión de estos deseos podrían, tras una terapia psicoanalítica exitosa, articular los propósitos que sus síntomas expresan. El obsesivo podría decir, solo después de ser iluminado por el psicoanálisis, algo así como que su razón para lavarse las manos repetidamente es "limpiar las manchas de los malos pensamientos de índole sexual que me persiguen sin descanso". El masoquista podría explicar también, y solo después de un proceso terapéutico que cumplió sus objetivos, que su compulsión a castigarse era una manera de aliviar un sentimiento de culpa. En el paradigmático caso que presenta en ese ensayo, el de la mujer que realizaba el ritual de manchar un mantel con tinta roja y llamar a su criada para que vea la mancha, la paciente también podría decirnos nuevamente, solo si gracias a la cura psicoanalítica accede a sus verdaderos motivos, que la finalidad de 
su acción era combatir la vergüenza suscitada por su frustrante noche de bodas ${ }^{4}$. En el razonamiento que ha llevado a realizar este tipo de acciones, hay siempre un paso que podemos llamar defectuoso y que generalmente son las premisas. Pero en todos estos casos, se trata de acciones que persiguen un fin que es visto por la persona como apropiado, bueno, deseable, y eso fue advertido por Freud.

Además de no considerar este aspecto de los escritos de Freud, también se le debe reprochar a Maclntyre el no tomar en cuenta que los deseos inconscientes, por ser inconscientes, actúan de un modo parecido a las causas y que, por lo tanto, no puede separarse (tan claramente como él lo hace) el elemento causal del elemento intencional en los motivos inconscientes. Esto es así porque las razones que forman los motivos inconscientes no actúan solo como razones. Una razón es, por definición, un argumento que puede ser criticado y contrastado con otras razones. Quizá podríamos decir que una razón es un argumento que ha sido revisado y comparado con otras razones. Eso no pasa con las razones que actúan desde el inconsciente. Precisamente por ser inconscientes, han eludido esta comparación con otras razones. Por eso tienen una fuerza causal y suelen estar al servicio de la irracionalidad, si cabe el oxímoron. Estas razones empujan, la mayoría de las veces ${ }^{5}$, a hacer cosas que desde la perspectiva del observador son compulsivas, erráticas, emocionalmente costosas y en muchos casos poco menos que inútiles. Tanto Jürgen Habermas como Donald Davidson han elaborado ingeniosos esquemas para tratar de entender cómo los motivos inconscientes, sin dejar de ser razones, son también causas.

En la segunda edición de su libro, MacIntyre (2004) reconoce que no le prestó suficiente atención al "deseo" y sí a las razones, y que las motivaciones inconscientes actúan de un modo distinto a las razones conscientes. Esto es una manera de admitir que cuando se habla de deseos inconscientes no puede hacerse una distinción tan contundente como la que él hace entre causas y razones. A pesar de este problema, esta primera parte de la tesis de Maclntyre es útil en la medida que enfatiza el aspecto teleológico de los impulsos inconscientes y combate de este modo una tendencia "biologizante" todavía existente en ciertos círculos psicoanalíticos.

La segunda parte de la crítica de Maclntyre a Freud es sobre la verdadera naturaleza del factor causal en las explicaciones psicoanalíticas y sobre la utilidad, o inutilidad, del concepto de inconsciente para estas explicaciones. De acuerdo a The Unconscious, no son los impulsos o los motivos los que tienen una fuerza causal, sino más bien las

4 Su acción buscaría generar la creencia de que su marido no había sido impotente en la noche de bodas.

5 La mayoría de las veces porque, como lo demostró Robert Audi, hay motivos inconscientes que son racionales. Un acto fallido puede ser, en algunas circunstancias, la respuesta más racional de una persona ante una situación. 
experiencias traumáticas de la infancia sedimentadas en la memoria las que actúan como gatilladores de esos impulsos. De aquí Maclntyre concluye, en lo que es la tesis más provocadora de su trabajo, que para explicar esta relación de causalidad entre experiencias infantiles y acciones en la adultez (y, por lo tanto, para predecir con rigor científico las conductas) no necesitamos el concepto de inconsciente.

De acuerdo a la interpretación que hace Maclntyre de los textos de Freud, hay un factor causal en las compulsiones neuróticas que se encuentra entrelazado, confundido, con los propósitos o motivos inconscientes. Este factor causal se origina en experiencias traumáticas en la infancia. Son estas experiencias, que quedan grabadas en la memoria con una carga emocional angustiosa, las que sirven como una especie de catapulta de los motivos, o razones, como los arriba descritos. Estos motivos son, por lo tanto, "defensas" contra el dolor, huidas frente a la angustia. En ellos radica el elemento intencional enfatizado por Maclntyre. Estos motivos necesitan, para existir, de ese sedimento de experiencias que actúan como una causa. Para respaldar su interpretación, Maclntyre se refiere al caso de la mujer de 19 años que ejecuta rigurosamente un complicado ritual antes de dormir, que Freud reporta en el mismo artículo "El sentido de los síntomas" (1917). Se trata de un ritual obsesivo que tiene la finalidad de aminorar la ansiedad. Esta ansiedad en relación con el dormir (el elemento causal para Maclntyre) se originó en una experiencia infantil que Freud solo insinúa y que parece haber sido el descubrir el coito de sus padres. En la vida adulta habrá situaciones que evoquen esa angustia y, aunque la paciente no podrá recordar la experiencia ni entender el objeto de esa ansiedad, esta será la que impulsará causalmente las maniobras defensivas que, en su caso, son los complicados rituales obsesivos. Así construye Maclntyre la conjunción entre un poder causal y una razón en las acciones neuróticas. Según el autor, podemos diferenciarlos con claridad porque son lógicamente independientes. Lo causal está en las situaciones traumáticas y su presencia afectiva, lo intencional, en las conductas a través de las cuales la persona quiere disminuir la ansiedad.

Maclntyre cree, y esta es la tesis central de su libro, que para entender este aspecto causal en los deseos de los neuróticos no es necesario el concepto de inconsciente. "La psicología tiene una deuda inconmensurable hacia Freud por haber sugerido tan claramente esas correlaciones, pero no hay nada particularmente freudiano acerca de ellas" (MacIntyre, 2004, p. 94). "Las hipótesis de Freud sobre el origen infantil de los rasgos adultos y de los desórdenes pueden formularse sin hacer referencia alguna al concepto de inconsciente" (MacIntyre, 2004, p. 97). En otras palabras, no hay nada particularmente freudiano en establecer esta relación causal entre experiencias tempranas y rasgos de la personalidad adulta. La psicología puede investigar esta relación del mismo modo como se debe investigar cualquier otra relación causal entre variables. En estas investigaciones, el concepto de inconsciente no tendrá ningún rol explicativo. 
Por esta razón, concluye MacIntyre, siempre que se usen como adjetivo o como adverbio los términos inconsciente o inconscientemente, estos son útiles porque permiten una descripción más fina del comportamiento: “Él [Freud] introduce inconsciente como un adjetivo para describir lo que hasta ahora habíamos observado, pero no habíamos reconocido ni clasificado" (Maclntyre, 2004, p. 78). Pero cuando Freud usa el término inconsciente como un sustantivo, esto implica que estamos considerando al inconsciente como un concepto cuya justificación son sus efectos causales. Y lo que no puede ver Maclntyre es cuáles son esos efectos y cuál es, por lo tanto, la contribución a la ciencia de los conceptos de inconsciente y represión para entender la relación causal entre experiencias infantiles y personalidad adulta.

\begin{abstract}
Mientras que Freud iluminadoramente presenta una buena cantidad de comportamientos como motivados inconscientemente y describe también cómo el recuerdo de ciertas situaciones y eventos que han devenido inconscientes puede tener un efecto terapéutico, él (Freud) desea justificar no solo el adverbio o el adjetivo, sino también el sustantivo: el inconsciente. Pero de la suposición de esa entidad, ¿qué consecuencias emergen que no hubieran podido ser anticipadas sin recurrir a este concepto? (Maclntyre, 2004, p. 97)
\end{abstract}

No necesitamos el concepto de inconsciente para establecer las relaciones causales entre infancia y adultez. Maclntyre (2004) propone que la investigación empírica solo necesita establecer la variable o variables que se refieren a alguna experiencia infantil y, del lado de los supuestos efectos, los rasgos de la personalidad. La investigación nos dirá si las conjeturas se corroboran o se refutan. Es cuestión "de amasar evidencia" (p. 94).

Antes de intentar rebatir esta parte de la tesis de Maclntyre, es conveniente rescatar el elemento de verdad que existe en su reconstrucción de la teoría freudiana.

Es un hecho de la naturaleza que desde niños establecemos asociaciones entre el entorno y nuestros deseos, y que muchas de estas asociaciones se sedimentan y se convierten en valoraciones afectivas más o menos duraderas acerca de las cosas y las personas que nos rodean. Estas valoraciones son, como lo ha señalado una tradición que se remonta a David Hume y llega a Simon Blackburn y a otros autores contemporáneos, un elemento importante en la motivación de nuestras acciones. Estas asociaciones afectivas se generan mecánicamente, lo que quiere decir que no son la consecuencia de acciones intencionales. Por esta razón, el desarrollo en el individuo de estas valoraciones con poder motivacional se explica mejor en términos puramente causales. A este tipo de contenidos mentales debe corresponder lo que David Finkelstein (2003) llama brutes likes and dislikes, rasgos de la personalidad que no responden a una estructura cognitiva o proposicional, pero que son parte de nuestro caudal motivacional. Son proclividades afectivas que participan en nuestras deliberaciones prácticas. Si pensamos en el modelo deseo-creencia, es una parte importante del polo constituido por el deseo. 
Serán estos sedimentos emocionales el factor causal de algunas respuestas en la vida adulta. Freud dio cabida a este tipo de asociaciones contingentes en algunas de sus explicaciones. Por ejemplo, en el famoso caso "Juanito", nos dice que uno de los hechos que contribuyó a que Juanito desarrollara fobia a los caballos y no a otro animal es que él y su papá jugaban al "caballito" (Freud, 1926/1979b, p. 100).

Maclntyre tiene razón cuando dice que no hay nada particularmente freudiano en estas correlaciones y que pueden explicarse sin teoría específica. 0 quizá deberíamos decir, corrigiendo al autor, con un enfoque conductista. Nuestra vida está llena de actitudes, sentimientos y motivaciones que se han establecido como hábitos, y para muchos de estos hábitos no hay mejor explicación de su adquisición que la que sigue un modelo de condicionamiento. A través de las experiencias de dolor y de placer aceptamos ciertas cosas y rechazamos otras. Esas aceptaciones y rechazos son parte de nuestra manera de ser. Algunas de estas actitudes, por lo tanto, se originan con una participación nula o mínima de las creencias. Ellas son más bien generadoras de deseos y de creencias.

Pero Maclntyre injustificadamente generaliza esta verdad a todas las probables relaciones entre biografía y modo de ser. Incluso en los ejemplos que él considera, solo si la explicación es superficial, se puede prescindir del concepto de inconsciente. Por ejemplo, dice que la actitud hacia la madre está en algunos casos correlacionada con la actitud hacia la esposa. Por supuesto que una investigación sobre este hecho puede atender solo los aspectos más generales o superficiales de esta causalidad. Es probable que en muchos casos se obtenga lo que sugiere Maclntyre. Para establecer esta relación causal, que incluso podría aportar conocimiento, la noción de inconsciente es, en efecto, innecesaria. Pero una mirada más profunda, es decir, una mirada que capture más aspectos de esta relación causal, va a necesitar de conceptos psicoanalíticos.

El error de Maclntyre es no haber tomado suficientemente en serio lo que él mismo considera un triunfo del psicoanálisis. Porque una descripción mejor o más fina de las acciones de los seres humanos, como la que se obtiene gracias al psicoanálisis, no puede ser solo un logro estético, como equivocadamente pensó Wittgenstein y parece creer Maclntyre. En ciencia y en psicología, descripción y explicación son dos niveles del conocimiento. La explicación es un nivel superior en el sentido de que nos acerca más a la verdad. Pero una mejor descripción tiene que generar mejores explicaciones. Nuevas y mejores descripciones implican que hay una nueva y mejor explicación todavía no revelada; la misma cosa es vista ahora, con la nueva descripción, desde otro ángulo. Nuevas variables a tomar en cuenta aparecen. En toda mejor descripción hay, por lo tanto, una relación causal implícita que espera ser sistematizada.

Si dejamos esa mirada superficial y consideramos más aspectos del mundo interno de los individuos, como sus deseos y sus creencias, vamos a hacer inteligibles correlaciones entre biografía y personalidad que sin la idea de inconsciente permanecen 
incomprensibles. Algo similar dijo Freud cuando justificó el concepto de inconsciente. Lo que MacIntyre no tomó en cuenta, a pesar de que lo menciona en más de una ocasión, es que, en las acciones causadas por los contenidos inconscientes, la relación entre motivo y conducta no se manifiesta con la claridad con que lo hace en las acciones motivadas por razones conscientes. Precisamente, la categoría "inconsciente" es necesaria para explicar actos del habla que no son en un principio inteligibles. Esta es la idea implícita en la sugerencia de Wittgenstein sobre cómo definir lo inconsciente. En la comprensión cotidiana de las otras personas, y de nosotros mismos, usamos una gramática que confiere coherencia o racionalidad a las acciones. Desde esa gramática hay acciones que son ininteligibles hasta que introducimos el concepto de inconsciente. Pasa lo mismo con las relaciones causales entre experiencias pasadas y personalidad actual. No se entiende cómo puede haber una relación causal entre, por un lado, un padre muy severo -y aun abusivo - con su hijo y una actitud amorosa del hijo hacia ese padre, hasta que el concepto de una formación reactiva inconsciente vuelve inteligible esa respuesta. Parece incomprensible que una persona que creció con sentimientos intensos de envidia frente a un hermano tenga en su vida adulta, como un rasgo que define su personalidad, una actitud en extremo benevolente hacia ese hermano, pero esa actitud es más clara si sabemos que la idealización es un modo, inconsciente, de hacer menos dolorosa una historia de sentimientos de envidia. Los ejemplos pueden multiplicarse. El punto es que el concepto de inconsciente no solo hace más inteligible nuestra comprensión de las acciones contemporáneas de los seres humanos, sino también las relaciones entre biografía y personalidad.

Que Maclntyre, al parecer, no comprenda en qué consiste lo reprimido contribuye a que no vea la necesidad de introducir la noción de inconsciente para entender las correlaciones entre infancia y personalidad adulta. El filósofo critica que Freud utilice el término idea como lo usó John Locke, es decir, como una unidad discreta de vida mental. Sin embargo, él mismo cae en esa cosificación de lo mental cuando, en las posibles explicaciones de la influencia de las experiencias infantiles en la vida adulta, dice que busca, sin encontrarla, esa entidad que Freud llama inconsciente. La búsqueda de MacIntyre estaba, por lo tanto, mal concebida, porque no era una "entidad" lo que debía perseguir. Los contenidos inconscientes no son ni tan simples como la no muy clara categoría de “ideas" ni tan difusos como sería "la actitud hacia la madre". Los contenidos del inconsciente son actos del habla que fueron censurados y que han dejado una huella que se ha convertido en una parte de la personalidad. Son intenciones que no llegaron a cristalizarse porque el entorno no lo permitió. Como tales, lo reprimido o lo inconsciente son pensamientos complejos. Podemos decir, usando el lenguaje de la filosofía analítica, que lo inconsciente, al igual que lo consciente, se describe mejor como actitudes proposicionales. Lo inconsciente son creencias sobre cómo el infante percibe las cosas y deseos de cómo quisiera que fueran. En ese sentido, los contenidos del inconsciente apuntan a 
estados de cosas presentes, que pueden ser apreciados emocionalmente con placer o con dolor, y a estados de cosas futuros, que pueden ser temidos o deseados.

Algunos psicoanalistas han usado la palabra escena para explicar que lo reprimido son fantasías de situaciones que evocan una respuesta o un intento de respuesta por parte del infante. Otto Kernberg ha capturado también esta idea cuando dice que todo impulso está asociado a una imagen de uno mismo y una imagen del otro (el otro interiorizado, se entiende) unidos por un afecto.

Si se le entiende así, el concepto de inconsciente es un avance científico en la explicación de la conducta. Porque si consideramos estos actos del habla reprimidos en la infancia, podemos predecir o explicar consecuencias en la vida adulta que de otro modo permanecerían incomprendidas. Además, dado que se trata de relaciones causales que hay que poner a prueba, esta comprensión de lo inconsciente es la que permitiría al psicoanálisis responder satisfactoriamente a ciertas críticas referidas a su falta de rigor científico. El psicoanálisis debe plantear hipótesis que sean testeables, como lo señala la psicoanalista y filósofa Pushpa Misra, quien en su libro The Scientific Status of Psychoanalysis (2016) responde de manera plausible a las objeciones de Karl Popper, sobre la intestabilidad de las proposiciones psicoanalíticas, y de Adolf Grünbaum, en cuanto a su pobre poder predictivo. Las leyes científicas que surgirían de estas corroboraciones serían, por supuesto, menos estrictas que las que existen en las ciencias naturales. De un estilo parental, descrito con la suficiente complejidad, sin embargo, la teoría psicoanalítica puede anticipar ciertas consecuencias en la personalidad futura.

The Unconscious es el primer intento sistemático, plasmado en un libro, por interrogar a la teoría psicoanalítica desde las filosofías de Wittgenstein y de Gilbert Ryle. Es una obra que postula que las ideas de Freud sirven para narrar la vida de los seres humanos de un modo distinto, más agudo, entretenido y novedoso, pero no para explicar ni predecir las acciones de los seres humanos. He tratado de refutar esa aseveración y al mismo tiempo destacar los logros de este trabajo en acercar el psicoanálisis a la filosofía analítica de la mente.

Después de este libro, otros filósofos continuaron trabajando para ensamblar las enseñanzas de la filosofía de la mente, que se origina en Wittgenstein, con el psicoanálisis y con la idea de que las motivaciones inconscientes son aquellas que el agente no puede articular verbalmente. Jürgen Habermas y David Finkelstein son dos autores que han definido así las motivaciones inconscientes.

Las obras de Maclntyre, Habermas, Finkelstein y varios otros autores contemporáneos demuestran que construir puentes entre el psicoanálisis y la filosofía de inspiración analítica es un trabajo en curso y muy necesario para la tarea más general de completar una imagen científica del ser humano. 


\section{REFERENCIAS}

Anscombe, E. (1957). Intention. Harvard University Press.

Finkelstein, D. (2003). Expression and the Inner. Harvard University Press.

Freud, S. (1979a). Trabajos sobre metapsicología. En Obras completas (t. XIV). Amorrortu. (Trabajo original publicado en 1915).

Freud, S. (1979b). Inhibición, síntoma y angustia. En Obras completas (t. XX). Amorrortu. (Trabajo original publicado en 1926).

Honneth, A. (2009). Crítica del agravio moral. Fondo de Cultura Económica.

Kant, I. (1997). Foundation of the Metaphysics of Morals. The Library of Liberal Arts.

MacIntyre, A. (2004). The Unconscious. A Conceptual Analysis. Harvard University Press.

Misra, P. (2016). The Scientific Status of Psychoanalysis. Karnac.

Wittgenstein, L. (1953). Philosophical Investigations. Macmillan.

Wittgenstein, L. (1958). The Blue and Brown Books. Harper Torchbooks.

Wittgenstein, L. (1982). Conversations on Freud, Excerpt from 1932-1933 Lectures. En R. Wollheim y J. Hopkins (Eds.), Philosophical Essays on Freud (pp. 1-11). Cambridge University Press. 